\title{
Philosophiques
}

\section{Éros androgyne et logos philosophique}

\section{Josiane Boulad Ayoub}

Volume 12, numéro 1, printemps 1985

URI : https://id.erudit.org/iderudit/203274ar

DOI : https://doi.org/10.7202/203274ar

Aller au sommaire du numéro

Éditeur(s)

Société de philosophie du Québec

ISSN

0316-2923 (imprimé)

1492-1391 (numérique)

Découvrir la revue

Citer cet article

Boulad Ayoub, J. (1985). Éros androgyne et logos philosophique. Philosophiques, 12(1), 107-132. https://doi.org/10.7202/203274ar

\section{Résumé de l'article}

Après avoir précisé l'enjeu idéologico-politique que recèle la " perspective féministe » en tant que catégorie d'analyse appliquée à l'histoire de la philosophie et retracé les présupposés relatifs à la représentation de l'entreprise philosophique que ladite perspective enveloppe, communément parlant, on relancera la question ontologique du rapport entre le Logos philosophique, à ses débuts, et Éros, le démon originaire et dynamique du discours philosophique. Ce recours au Mythe aura fait émerger la figure originelle de la philosophie comme symboliquement androgyne (dialectique). On aboutira à la thèse d'une " perspective hermaphrodite " qui voudrait en finir - par la dialectisation opérée sur le mode plaisant — avec la catégorie " perspective féministe ", pour ce qui en est de son application à l'histoire de la philosophie.
Ce document est protégé par la loi sur le droit d'auteur. L'utilisation des services d'Érudit (y compris la reproduction) est assujettie à sa politique d'utilisation que vous pouvez consulter en ligne.

https://apropos.erudit.org/fr/usagers/politique-dutilisation/ 


\title{
ÉROS ANDROGYNE ET LOGOS PHILOSOPHIQUE
}

\author{
par Josiane Boulad Ayoub
}

\begin{abstract}
RÉSUMÉ. Après avoir précisé l'enjeu idéologico-politique que recèle la "perspective féministe" en tant que catégorie d'analyse appliquée à l'histoire de la philosophie et retracé les présupposés relatifs à la représentation de l'entreprise philosophique que ladite perspective enveloppe, communément parlant, on relancera la question ontologique du rapport entre le Logos philosophique, à ses débuts, et Éros, le démon originaire et dynamique du discours philosophique. Ce recours au Mythe aura fait émerger la figure originelle de la philosophie comme symboliquement androgyne (dialectique). On aboutira à la thèse d'une "perspective hermaphrodite " qui voudrait en finir - par la dialectisation opérée sur le mode plaisant - avec la catégorie " perspective féministe ", pour ce qui en est de son application à l'histoire de la philosophie.
\end{abstract}

ABSTRACT. To begin with, some of the ideological and political issues concerning the so called "feminist view" on the history of philosophy are brought forth. The ontological problem of the fundamental relationship between Logos and Eros is then analyzed, such as it occurs in the philosophical discourse of the beginnings. Finally, for a more dialectical approach than the traditional "feminist view", a-metaphorical androgynous view is suggested - with one's tongue in one's cheek ! - as an alternative thesis.

Dans les congrès de philosophie, l'interpellation de l'histoire de la philosophie apparaît indispensable : en effet, celleci, à la différence de ce qui se passe dans d'autres disciplines, se trouve liée organiquement au discours et à la pratique philosophiques ; dans un rapport d'introjection, ajouterait même le psychanalyste. Mais aussitôt une exigence méthodologique accompagnera cette interpellation : l'historien de la philosophie se réclamera de l'une ou l'autre des différentes perspectives possibles de la tradition philosophique, philologique, phénomé- 
nologique, herméneutique, marxiste ..., pour examiner sous leur angle le discours philosophique et en développer notre connaissance. Il va de soi que les catégories d'analyse employées devront révéler toute la richesse des nuances de l'histoire de la philosophie et faire résonner, chacune à sa manière, cette note juste de la différence philosophique dont parle Platon.

En revanche, convoquer la perspective féministe pour interroger l'histoire de la philosophie ainsi que le font de plus en plus souvent certains intitulés - en passe de devenir rituels de tables rondes ne va pas de soi. Intuitivement on pressent que se réclamer d'une telle perspective aura une signification à valeur politique et sociale avant d'être théorique. Le caractère insolite de cette catégorie d'analyse déconcerte ; même si l'on s'accorde à reconnaître le féminisme comme l'une des idéologies les plus pugnaces de notre culture, et partant, phénomène social digne d'intérêt pour l'interprétation philosophique, "l'inquiétante étrangeté " de la formule n'en demeure pas moins. Ne signalet-elle pas un coup de force dans la mesure même où elle se répand et devient pratiquement interrogation obligée ? Il ne s'agirait plus alors de disserter, à partir de la position méta ou épidiscursive, officiellement dévolue à la philosophie, sur un réel que ne penseraient pas habituellement les philosophes, ou auquel ils ne penseraient pas ; un renversement a été opéré : d'objet d'analyse le discours féministe commun, par la seule grâce de la formule, se retrouve sujet, critique du réel philosophique. Qu'on m'entende bien : je m'interroge ici sur le discours féministe commun ; ce dernier terme au sens idéologique : [cf. le concept du discours social commun (R. Fossaërt)] autrement dit, le discours féministe répandu, partagé, dominant, fonctionnant dans le texte social, comme discours idéologique dominant, partial, polémique, normatif, se manifestant dans des rapports de pouvoir et ayant un effet d'hégémonie. Mais précisément c'est bien cet investissement inopiné de la position métadiscursive, propre à la réflexion philosophique qui fait problème ; c'est bien cette créance conférée, du moins nominalement, au discours féministe commun d'interpeller l'histoire de la philosophie elle-même, tout comme les philosophies légitimes ou légitimées le font, à partir de leur perspective spécifique, et ce dans des appareils par excellence de l'Institution, les congrès ou colloques, c'est 
bien cela qui bouscule les règles du jeu. L'air apparemment innocent de la formule type, " perspectives féministes sur l'histoire de la philosophie ", ne devrait pas tromper : elle recèle un enjeu idéologico-politique qu'il importe de dégager puisque c'est cet enjeu qui lui donne sa signification profonde ; aussi bien elle soulève des difficultés d'ordre théorique et méthodologique qui devront être soulignées.

Dans un premier moment de mon exposé, je m'attacherai à préciser cet enjeu ainsi qu'à soulever quelques-unes de ces difficultés. On l'aura compris : répéter les « contenus» de la perspective féministe sur l'histoire de la philosophie m'intéresse moins qu'examiner le statut théorique de cette nouvelle " grille », sa pertinence eu égard à l'éclaircissement du discours philosophique et de son économie. Cependant, comme toute " perspective " enveloppe une certaine représentation de l'entreprise philosophique, je tâcherai, ensuite, de retrouver, d'une part, les présupposés relatifs à cette représentation et qui l'autoriseraient, d'autre part, le schème logique de la forme philosophique visée par ladite perspective, et qu'elle mettrait en cause : il s'agira, dans ce deuxième moment, d'analyser ce que "pointe " le discours féministe commun du discours philosophique. Il apparaîtra difficile d'esquiver la question métaphysique qui dès lors est relancée : qu'en est-il du rapport entre le Logos philosophique à ses débuts et Éros, le démon originaire et dynamique du discours philosophique ? Je tenterai, dans ce troisième et dernier moment de mon exposé, de caractériser la nature d'Éros Philosophe et de mettre en évidence quelquesunes des implications de sa nature duelle sur l'activité philosophique. Le recours au Mythe aura fait émerger une certaine figure de la philosophie. Figure originelle, celle-ci, symboliquement androgyne, se détache de l'bypokêimenon philosophique fondamental ; elle fait signe, en même temps, vers « ce qui demeure par dessous et repose au fond »; elle illustre les commencements du Logos philosophique de même qu'elle en inspire le déploiement : telle est la thèse que je soutiendrai dans cette portion finale de mon exposé. Son enjeu, par cette esquisse de la figure originelle de la philosophie comme figure androgyne, est d'en finir avec la catégorie " perspective féministe " pour ce qui en est de son application à l'histoire de la philosophie. Cette 
perspective aura été posée, puis niée ; l'aufhebung, si l'on peut dire, est tenté avec la « perspective hermaphrodite » que je propose : Éros Philosophe serait l'Éros Androgyne ; le discours philosophique se déploierait, au commencement et à la fin, sous l'horizon de ce « démon » qui insuffle la vie à son logos.

\section{1- PERSPECTIVE FÉMINISTE ET HISTOIRE DE LA PHILOSOPHIE.}

1.1 Proposer comme perspective sur l'histoire de la philosophie la perspective féministe soulève d'abord une difficulté d'ordre technique ou méthodologique sérieuse : il est évident que, si l'on décide d'interroger le corpus philosophique, cette enquête sera conduite en appliquant un modèle, ou du moins au moyen d'un ensemble de concepts permettant d'analyser, ou encore de mettre en évidence, les caractéristiques du discours philosophique, ses problèmes spécifiques, ses méthodes particulières, ses opérations propres, ainsi de suite. Il est non moins évident que l'historien de la philosophie empruntera pour sa démarche, soit un modèle déjà éprouvé et dérivant de la tradition philosophique elle-même, soit proposera une nouvelle méthode qu'il aura pris soin de fonder, ou dont il aura pris soin de légitimer la validité, selon les critères scientifiques en usage. Dans l'un ou l'autre cas on peut dire qu'il s'agira d'un discours du même au même : un redoublement - le plus souvent explicite - du discours philosophique; redoublement non seulement effectif, mais qui plus est, apparaîtra, pour l'historien de la philosophie, sinon l'une des conditions déterminantes de son travail, comme sa finalité, afin que celui-là ne soit pas simple reproduction mais production philosophique originale. La démarche de l'historien de la philosophie, dont l'objet est le discours philosophique lui-même dans son ensemble, tend à se confondre avec celle du philosophator proprement dit, qui étudie des objets particuliers, le discours des sciences par exemple. C'est une démarche analytique, et normative la plus souvent, un entêtement patient et méthodique dans l'éclaircissement de l'objet étudié, une entreprise au premier chef métadiscursive et qui tend à constituer une théorie de . . . théorie de la philosophie, théorie de l'idéologie, théorie des systèmes, somme toute une langue dans une langue. Or le discours féministe soulève, eu égard au discours de l'histoire de la philosophie, deux questions au moins, 
qui, à ma connaissance, n'ont pas été sérieusement prises en charge par ce premier : à quelles conditions le discours féministe pourrait-il être un discours du même au même dans le sens évoqué ci-dessus ? La seconde question est moins ambitieuse mais peut-être plus exigeante : à quelles conditions le discours féministe serait-il susceptible de produire des outils d'analyse efficaces pour mettre en évidence les caractéristiques de l'activité philosophique?

Ces deux questions nécessitent un travail préalable de réflexion sur le travail philosophique ou du moins d'explicitation des hypothèses directrices de la perspective adoptée dont précisément les tenants de la perspective féministe ont semblé jusqu'ici faire l'économie. Et cela se comprend, dans une certaine mesure, puisque lesdits champions pourraient répondre à peu près dans ce sens à la première question : par définition, le discours féministe sur le discours philosophique ne tend pas à se constituer én redoublement (ou dédoublement) de celui-ci mais, au contraire, il ne surgit qu'à la faveur d'un mouvement d'extériorité qui le situe dans une altérité radicale vis-à-vis du discours philosophique. Pour la seconde question, on est obligé de constater non pas qu'elle ne préoccupe guère les tenants de la perspective féministe, mais, plus fortement, qu'elle ne les intéresse tout simplement pas. Si, par définition, le discours féministe est le discours de l'Autre, ou du moins, si on atténue cette position, s'il constitue le discours de l'Étranger (ère), son « zèle pour la philosophie (n')est (pas) grand » pour autant, tout au rebours de ce que dit Théodore dans le Sophiste pour présenter à Socrate et à ses amis l'étranger venu d'Élée, qui sera invité à discourir sur les distinctions à faire entre sophiste et philosophe. La logique - téléonomique - de la perspective féministe est ordonnée à ses propres fins ; pour paraphraser encore Platon, (lettre VII, $341 \mathrm{c}-\mathrm{d}$ ) on pourrait écrire : la perspective féministe ne produit pas un savoir qui, à l'exemple des autres, puisse aucunement se formuler en propositions ; mais résultat de l'établissement d'un commerce répété avec ce qui est la matière même de ce savoir (la condition féminine sans doute ?), résultat d'une existence qu'on partage avec elle, soudainement, comme s'allume une lumière lorsque bondit la flamme, ce savoir se produit dans et par la perspective elle-même, et désormais, il 
s'y nourrit tout seul lui-même. Autrement dit, la perspective féministe ne se constitue pas dans un effort cognitif vis-à-vis d'un autre discours, ou encore, ne se place pas sous l'horizon du discours philosophique considéré en tant que tel : elle ne tend qu'a montrer la " culpabilité » du discours philosophique, entendre, son silence conceptuel, son refus de penser la réalité de la femme, et aboutit ainsi dans le renvoi à elle-même, et à la consolidation de sa propre perspective comme la seule judicieuse. Par ailleurs, a-t-on jamais défini les caractères propres d'une perspective féministe et ce qui la distinguerait d'une perspective phénoménologique, par exemple ? Il y a fort à parier que ce débat, s'il s'est jamais tenu, ne se livrera pas sur le terrain gnoséologique, mais axiologique, ontologique, praxéologique ou encore politique. Je ne soutiens pas ici que ces divers et derniers terrains n'ont pas leur intérêt, je fais simplement remarquer que la perspective féministe n'a pas encore, ni clairement délimité son propre terrain, ni envisagé les implications méthodologiques cohérentes avec le choix de son ancrage méthodologique. Les difficultés ne rebondiraient-elles pas, par ailleurs, car pourrait-on caractériser - objectivement - et sans recourir à des métaphores ou à des analogies fragiles, une écriture philosophique féministe et une écriture philosophique masculiniste?

1.2 En second lieu, d'un point de vue plus strictement théorique cette fois, la perspective féministe soulève encore une autre série de difficultés. Si, comme nous l'avons dit, la perspective féministe n'est somme toute intéressée qu'à elle-même, et que son objet conjecturel ne lui importe guère en tant qu'objet mais seulement dans la mesure où il est un prétexte à établir son contexte, sa position par opposition, le mouvement qui l'anime ne serait-il pas circulaire, pour ne pas dire spéculaire? Le corpus que scrute la perspective féministe, qu'il relève de l'histoire de la philosophie, des sciences, de la religion ou de la société, semble lui faire office de miroir ; elle consulte ce corpus, à la manière pour ainsi dire de la marâtre de Blanche-Neige consultant sa psyché : miroir, miroir, dis-moi si je suis belle, dis-moi si je suis la plus belle ! le corpus étudié ne le sera qu'en fonction de qui l'étudie, dans un renvoi perpétuel à l'élucidation de la perspective qui l'envisage. Paradoxalement, l'analyste 
féministe, Narcisse d'un nouveau genre, n'est jamais plus aise que lorsqu'il ne découvre pas son reflet là où il se mire, mieux encore lorsque ce reflet est déformé : miroir, miroir, dis-moi si je suis la plus laide ! Nous touchons là, devant cet effet, appelonsle paranarcissique, à l'enjeu fondamental du discours féministe, avant tout idéologico-politique. Feignons un moment de ne pas l'avoir aperçu et poursuivons la chasse encore un peu. De quelle sorte d'efficacité s'agit-il lorsqu'une perspective, qui, par définition, doit nous faire apercevoir ce qui s'étale devant soi et nous permettre de le cadrer, ne genère qu'un sens obligatoire - tous les autres étant sens interdits -, auto-référentiel ? Quelle belle chose que la perspective ! s'exclamait je ne sais plus quel peintre italien en parlant, bien entendu, de la perspective au sens de cette technique, alors nouvelle, de la représentation picturale. Dans le cas de la perspective dite féministe, tout se passe comme si c'était ce seul sens de perspective qu'on aurait en vue ; autrement dit, on s'extasie devant la promesse qui semble représenter le seul fait de la montée en scène de la perspective féministe sur . . . le constat ou le rappel de cette " audace » se substitue à l'établissement précis du renouvellement significatif qu'elle permet sur . . . ou du progrès de l'interprétation qu'elle autorise sur . . . Le même phénomène jouerait du reste pour la perspective des autres discours dominés, théoriquement et politiquement parlant, juif, noir ou gai. Mais alors serait-ce à dire que lesdites perspectives ne comptant ainsi que sur des effets auto-fondationnels et auto-référentiels, auraient un statut à dominante idéologique ? Leur intérêt, avant tout émancipateur, détermine le choix du primat qu'elles accordent aux critères de la pratique sur ceux de la raison théorétique, et n'interdit pas, dès lors, la hiérarchisation d'ordre axiologique ou politique qui est inévitablement introduite du même coup ${ }^{1}$. C'est dire que nous sommes non pas dans une perspective aléthique mais, au meilleur, kaïrotique, (on sait l'importance qu'accorde la sophistique à la notion de kaïros, du moment opportun, qui anime sa logique pragmatique), au pire, «moraliste ». Comme dirait

1. Au point de vue épistémologique, Platon nous rappelle déjà dans le Sophiste que " pour suivre la piste qui est celle des notions, l'art de frotter avec l'éponge n'est nullement moins ni non plus davantage intéressant que celui d'administrer une potion médicinale . . . il sera complètement indifférent pour notre démarche de savoir quelle dénomination sera jugée la plus décorative ... Sophiste, $228 \mathrm{c}$. 
cette fois Althusser, et non Platon, ce qui se passe avec la perspective féministe, la signification de cette mise d'un discours en position de domination ou de transcendance, de cette mise au pouvoir discursif, est politique ; elle n'a pas d'objet mais un enjeu : le pouvoir et un objectif : les effets du pouvoir. Confondant délibérément lutte des sexes et lutte de classes, la perspective féministe sur l'histoire de la philosophie se veut en dernier ressort lutte de classes dans la théorie, une guerre politique continuée par des moyens théoriques. On pourrait malicieusement rectifier : lutte de places dans la théorie.

1.3 Ce qui nous conduit à examiner maintenant l'enjeu fondamental de la perspective féministe sur l'histoire de la philosophie, la charge politique qui le leste et qui lui donne sa véritable signification d'ordre polémique, celle d'une lutte idéologique venant redoubler, au plan symbolique, non pas le discours philosophique mais bien un des aspects du discours féministe et de la lutte politique qu'il a entreprise dans nos sociétés.

La perspective féministe sur l'histoire de la philosophie renvoie donc au discours féministe tout court. Or celui-ci préconise, on le sait, l'extension du rôle et des droits de la femme dans la société. Discours de lutte, aux visées révolutionnaire et progressiste, il cherche à mettre en place les conditions politiques, idéologiques et économiques de la transformation sociale : dès lors la perspective féministe sur l'histoire de la philosophie, ou sur l'histoire de la musique, ou sur l'histoire des mathématiques, en tant que constituant un discours de lutte idéologique, fonctionnera selon un schème réglé par un double mouvement :

a) la dénonciation, dans les histoires particulières des disciplines, de la répétition de l'absence des femmes ou de l'occultation de leurs problèmes et de leur réel. Cette répétition - dénonciation des histoires particulières qui dédouble celle de l'Histoire, est elle-même répétée dans la tradition du discours féministe mais rarement éclaircie, et pour cause : l'effet cherché n'étant pas d'expliquer par le contexte culturel ou historique la situation particulière du discours dénoncé, mais de reconstruire l'histoire selon une gynodicée, si je peux m'exprimer ainsi, normative comme toutes les logodicées. 
b) la revendication de la présence des femmes ou encore de la non-occultation de leurs problèmes, des thèmes qui leur sont propres, de leur réel.

Généralement parlant, c'est le moment de la revendication qui constitue le moment fort du discours : il surdétermine le moment de la dénonciation et lui donne son sens, tétrospectivement parlant. Le moment de la dénonciation n'étant là, non pas pour des fins " historiques », d'où, du reste, les compilations hâtives et souvent erronées ou encore partiales qui marquent le plus souvent ce moment, mais plutôt pour " creuser " le terrain, le vider à l'image de l'absence dénoncée : ainsi la présence que l'on revendique apparaît légitime, nécessaire ; elle vient non pas remplacer quelque chose, non pas se substituer à quelque chose, mais bien occuper, de plein droit, logique et historique, un vide. Le ressort ontologique traditionnel a joué : le postulat, l'Être est préférable au Non-Être, s'accompagne de la relation, préparée dans le premier moment, qui est établie entre, d'une part, être et vérité (présence) et, d'autre part, nonêtre et erreur (absence). L'équation ontologico-gnoséologique traditionnelle autorise ainsi (fonde ?), en tout cas, rationalise, l'enjeu idéologico-politique.

Tenir une perspective féministe sur l'histoire de la philosophie, revient, en dernier ressort, et en cohérence avec les postulats impliqués, à se faire l'écho discursif de la lutte sociale des femmes et à traiter l'histoire de la philosophie comme un moyen de cette lutte mais non comme une fin ; le souci cognitif devra s'effacer devant l'impératif pragmatique. Une action - mais la perspective est-elle une action? - plus féconde, plus créatrice, et à la longue plus efficace, semblerait être celle-ci : se mettre à l'ouvrage, et accordé qu'une typologie de féminin ait été établie, écrire philosophiquement-féministiquement, ou encore, et peut-être moins ontologiquement, écrire avec, et selon, les thèmes, les problèmes du réel féminin. Et ici Simone de Beauvoir est exemplaire. Autrement dit, créer, produire de la philosophie à partir d'une prise de position théorique lucide et explicite ; construire, de l'intérieur, son discours philosophique distinctif, non plus vilipender, de l'extérieur, le disco: rs de la philosophie éternelle. Platon divisait les arts entre arts d'acquisition et arts de production. Il semble urgent que le discours 
féministe se presse de rejoindre - philosophiquement à sa façon - la deuxième catégorie et cesser de se cantonner, comme il l'a fait largement jusqu'à présent, dans le stade de la négation, de la critique négative.

Il est juste pourtant de se demander si cette dernière attitude est dictée seulement par les enjeux du discours féministe ainsi que je les ai envisagés dans cette première partie, ou bien si, plus radicalement, la nature même du discours philosophique y serait peut-être pour quelque chose. Ceci nous ramène au cœur du malentendu qui me semble exister dans les rapports entre femme et philosophie. Il serait opportun de scruter maintenant les ombres et les lumières du discours philosophique tel que le partage le discours féministe.

\section{DISCOURS PHILOSOPHIQUE ET DISCOURS FÉMINISTE.}

Pour essayer de déterminer ce que je crois être les présupposés du discours féministe commun sur le discours philosophique, présupposés qui servent de base à la représentation féministe du discours philosophique, mieux vaut repartir de Platon, lui qui commence la philosophie. C'est du Sophiste que je prendrai mon élan, de ce dialogue qui pose la question d'origine (de la philosophie), la question d'identité (de la philosophie) comme liées l'une à l'autre et inséparables, en même temps, de la question métaphysique, de la question de l'Être. On remarquera que j'inverse, dans cette étape, la perspective : je pars, maintenant, du discours philosophique pour essayer de découvrir ce qui a pu y donner prise aux imputations du discours féministe et informer les présuppositions initiales de ce dernier.

2.1 La philosophie commence par le parricide. Dans sa chasse à l'Être, l'Étranger déclare, par trois fois, à Théétète, son interlocuteur, du Sophiste, qu'il se voit contraint à cette " audace » théorique s'il veut échapper à l'impasse logique où l'a conduit, à cette étape de son argumentation, l'enseignement de son maître d'Élée. Il leur faudra donc transgresser l'interdiction parménidienne : penser «que ce qui n'est pas est "; autrement dit, s'efforcer dans cette seconde partie du dialogue, de penser ensemble l'être et le non-être. C'est alors qu'on pourra définir la nature de l'Être par la participation mutuelle des Formes ou la com- 
munication des genres ${ }^{2}$. L'enjeu de ces analyses portant sur les réalités génériques premières, le Mouvement, le Repos, le Même, l'Être, l'Autre auquel sera ramené le Non-Être, sur leurs rapports ainsi que sur les lois qui gouvernent ceux-ci, est en effet d'importance : ce travail, à la fois "difficile et beau ", comme le dit Platon, servira à faire comprendre, d'une part, la nature vraie de la pensée concrète, du jugement et du discours (et la privation du discours ferait que la Cité serait privée de philosophie, puisque c'est le discours qui monnaye ici-bas les réalités génériques (Sophiste 260 a), d'autre part, la fonction de la dialectique et de la philosophie. L'Être, région à l'éclat éblouissant est le lieu de la philosophie et de la vérité de son discours. La connaissance, cet Éros, ce désir qui anime l'homme libre, le philosophe à proprement parler, ne s'obtient que par la compétence dialectique, l'art que seul le philosophe exerce de façon "pure et légitime " : "diviser selon les genres et ne point juger le même une nature qui est autre . . . et cela (discerner comme il faut) . . . aussi bien selon la manière dont les natures peuvent communiquer que selon la manière dont elles ne le peuvent pas, c'est savoir discriminer selon le genre " (Sophiste $254 \mathrm{e}$ ). Il s'agira donc pour la philosophie et pour son exercice dialectique de s'ancrer dans l'Être ; le travail de purification que lui reconnaît en propre Platon consiste en un triage d'ordre conceptuel. En revanche, le Non-Être, région où règne une obscurité aussi profonde que l'était celle de la lumière baignant la région contemplée par le philosophe, est le lieu où s'enfonce son rival discursif, le Sophiste. Celui-ci a « pris subrepticement la fuite vers l'obscurité du Non-Être, se l'attachant à lui-même à force de le pratiquer ; homme que l'obscurité du lieu rend difficile à observer par l'esprit » (Sophiste 254 a).

2. "Les genres . . . se mêlent entre eux : L'Être et l'Autre circulent à travers tous et ces deux genres à travers l'un l'autre ; l'Autre participant à l'Être « est ", non qu'il soit cependant ce dont il participe, mais autre chose, et, d'autre part, étant autre chose que l'Être, forcément il est en toute certitude non-être. Quant à l'Être, puisqu'à son tour il participe à l'Autre, il doit être autre que le reste des genres ; mais puisqu'ainsi il est autre que tous les genres sans exception, il n'est pas chacun d'eux, pas davantage il n'est l'ensemble de ces autres genres, réserve faite de ce qu'il est lui-même ; par suite sans contestation possible, l'Être à son tour, milliers de fois sur milliers de fois n'est pas, et c'est ainsi dès lors que, hors lui, tout le reste aussi bien pris individuellement que dans son ensemble, un grand nombre de fois " est ", un grnd nombre de fois d'autre part "n'est pas " ". Sophiste $259 a-b$. 
2.2 Je disais tantôt que les thèses sur la philosophie soutenues par Platon au bout de son questionnement sur l'Être me semblaient propices à éclairer, de l'intérieur même du discours philosophique, le contexte de la représentation idéologique élaborée par le discours féministe commun. Aussi ai-je commencé par rappeler les propositions platoniciennes et les démarcations qu'elles établissent afin de pouvoir en dégager les implications, sur le plan idéologique, qu'en tire le discours féministe. Je suppute, en effet, que c'est au discours philosophique fondateur que s'alimentent les énoncés courants du féminisme relatifs à la situation de la Femme dans la philosophie et à la caractérisation subséquente du discours philosophique comme discours de dénégation de la Femme ou de son assujettissement. J'avancerai que sur le plan de son rapport au discours philosophique, tout se passe comme si le discours féministe commun ou plus précisément l'objet de ce discours, la Gunê, la situation qui lui est faite, est identifié au Sophiste - j'entends, à sa figure transhistorique. Par suite le discours féministe s'emploiera à dénoncer la triple distinction qui sépare, à son point de vue, le Philosophe de la Gunê ; de plus, dans ces distinctions, le discours féministe voit une manœuvre, un stratagème du discours philosophique pour accomplir la dissolution théorique de la Femme, ou du moins sa dénégation.

Le topos où se tient le Sophiste - Gunê est celui du NonÊtre, du monde sensible, des corps ; du fait même il est séparé du topos où s'élance, par son affinité avec le divin, l'esprit du philosophe : l'Être ou le monde intelligible. La seconde distinction est celle qui est faite eu égard à la situation sociale, métaphore politique de la situation théorique, des personnages en question : le philosophe est l'homme libre à tous égards, le Citoyen par excellence, l'aristocrate de la pensée, celui qui légitimement est invité « à voir ", à connaître alors que le SophisteGunê serait le non-libre par excellence, l'esclave ou le métèque, voué au train-train de la vie quotidienne, à la pratique ou à l'exercice rétribué. Enfin la connaissance propre à l'homme libre, au philosophe s'adresse aux concepts, à l'universel, suppose le travail de production de l'eikastikê, la copie fidèle de la réalité, et partant le simulacre vrai. C'est ainsi que le philosophe est le savant suprême, celui qui atteint le degré supérieur du Monde 
Intelligible, le domaine de l'epistême, et se distingue du SophisteGunê qui ne produisant que le phantastiké, le simulacre menteur, l'illusion, se complaît dans le particulier, dans le Monde Sensible, et dans la manipulation de la doxa.

Le discours féministe se représentera donc le discours philosophique comme se constituant par division et hiérarchisation, ne s'attachant que ce avec quoi il a communauté et excluant, ou du moins donnant préséance à, certaines notions et à certains thèmes sur d'autres, rejetant du côté de l'Autre ce qui n'est pas le Même. La Gunê se retrouvant chaque fois, sous couvert d'opérations logiques, qu'elles soient menées du point de vue de l'extension du genre, de la compréhension, de la subordination ou de la coordination, du côté de l'Autre, hors philosophie.

2.3 On peut inférer maintenant de cette représentation, laquelle à son tour anime les prises de parti vengeresses du discours féministe commun à l'égard de la philosophie, sa présupposition fondamentale. De nature ontologique, celle-ci formulerait que l'être féminin, le réel féminin, constitue, du point de vue de la philosophie, un non-être. On tient ici, croirais-je, la racine du malentendu qui s'est perpétué au sujet du rapport femme-philosophie. On se rappellera pourtant, et tel était l'enjeu du parricide, le fait que le non-être ne peut s'envisager que dans son rapport à l'être ; c'est dire qu'il est d'une certaine façon, qu'à proprement parler il constitue l'Autre de l'Être : "L'Être et l'Autre circulent à travers tous et ces deux genres à travers l'un l'autre. L'Autre participe à l'Être, est . . . quant à l'Être, puisqu'à son tour il participe à l'Autre, il doit être autre que tous les genres . . . par suite . . . l'Être à son tour n'est pas . . .». À suivre Platon, le Sophiste-Gunê ne doit ni ne peut être séparé du Philosophe : «milliers de fois sur milliers de fois " l'échange a lieu au sein de l'Être. La Gunê est, participe au Philosophe et, à son tour, le Philosophe participe à la Gunê. Le discours de la philosophie se construit avec et selon le discours de l'Autre, il est toujours en relation avec lui, son opposé le plus souvent, mais jamais son contraire. C'est dans cette dynamique incessante que le discours progresse, se renouvelle et surtout s'unifie. Sa logique ne saurait exclure, dénier ce qui, au contraire, relance perpétuellement son mouvement. L'Autre, la Gunê, circule en 
"quantité infinie " dans la trame de l'Être où se tissent les fils entrelacés du discours philosophique.

2.4 Le discours féministe, dans sa mise en cause politicoidéologique, c'est-à-dire polémique, du discours philosophique, a préféré confondre le non-être avec le néant, et non l'expliciter comme l'Autre joint à l'Être. Il s'est mépris, par conséquent, sur la situation de la Gunê par rapport au Philosophe ; il l'a vue séparée de ce dernier, exclue ou assujettie par lui. Et même si quelquefois le discours féministe a accepté de poser Gunê comme l'Autre du Philosophe, il n'a pas retenu pour autant la leçon dialectique de Platon qui établissait le schème de relation entre les essences ou les discours. Le discours féministe a dénoncé le discours philosophique comme enfermant une infinité de néants, les néants de ce que ce discours n'est point ; il n'a eu de cesse alors de nommer et de pointer dans le discours philosophique quelques-uns de ces néants auxquels Gunê s'identifie et qui la déterminent comme exclue du discours philosophique : non pas l'Autre, mais l'Absente en quantité infinie, et dans le même mouvement, l'Être, dont se réclame le discours philosophique, sera qualifié comme l'éternel Présent qui justifie la dominance se voulant éternelle de l'Anêr-Philosophe. De même que la philosophie serait largement encore, historiquement parlant, le fait masculin, de même, théoriquement parlant, les concepts, les thèmes qu'elle exploiterait, les thèses qu'elle avancerait lui conféreraient, si le concept même de la philosophie se formule 'et se précise en même temps que l'histoire de la philosophie, son caractère phallocratique et la déconsidération qui entoure les notions qui nouent le réel féminin : préséance de la " virilité " du conçu par rapport au vécu (rattaché au "féminin "), pouvoir de l'abstrait par rapport à la vitalité immédiate ou encore silence sur les raisons du corps, sur les forces du désir, tout ce qui éclairerait la réalité des rapports concrets homme-femme.

Le schème du Même et de l'Autre règle la logique du discours philosophique. Le malentendu a surgi dans la méconnaissance, d'une part, de cette relation dialectique entre les termes prise comme une simple alternative, d'autre part, sur l'identification, qui se serait actualisée dans l'Histoire, du Même (la philosophie en tant que telle) avec l'Anêr, de l'Autre avec la Gunê. Le régime discursif de la philosophie soumis aux con- 
traintes sociales, culturelles, historiques n'aurait procédé que d'un parti-pris masculin, privilégiant de la sorte certaines valeurs ou notions dites viriles sur les autres dites féminines. Ainsi le discours historicisé de la philosophie éternelle, serait celui du discours de l'Homme, de l'Anêr ; prétendant rendre compte de toute la réalité il laisserait échapper la réalité propre à la sophistique éternelle, occulterait sans cesse la Femme, la Gunê. Voici, croirais-je, l'essence du malentendu, malentendu qui repose en substance sur l'appréhension du logos philosophique selon un certain mode : l'écartèlement irréductible de ses éléments. La vision féministe courante fait, en définitive, du Logos philosophique, un Logos à jamais déchiré. Je ne tiens pas cette vision pour irréfragable. Pour la dépasser et ramener peut-être « la paix perpétuelle ", comme dirait Kant, entre perspectives à valence "politique ", je voudrais maintenant gagner avec une disposition gaie et ludique le domaine du Mythe, et proposer une perspective qu'on pourra qualifier, abstraction faite de toute sexualisation, d'hermaphrodite.

\section{3. ÉROS ANDROGYNE ET PHILOSOPHIE.}

3.1 Thanatos se profile aux commencements de la philosophie, disais-je tout à l'heure ; c'est bien peut-être le dieu ainsi que les métonymies qui l'escortent qui commandent son départ ; elle se déploie ensuite dans sa marche vers la connaissance, au rythme de l'entrelacement du Même et de l'Autre. Il ne faudrait cependant pas oublier, si l'on veut compléter la fresque mythologique, d'invoquer également la force dynamique qui soulève et anime l'élan de la recherche de la vérité, Éros, le dieu - plus justement, le démon - quasi éponyme de la philosophie. Érôs, présent, enchâssé dans son nom, dès sa naissance : passion, amour, désir de la science, philosopher.

Le malentendu du discours féministe commun à l'égard de la philosophie reposait, avançais-je, aussi bien sur son identification avec l'Autre, accompagné de l'interprétation de cet Autre comme Néant, que sur la méprise au sujet de la leçon platonicienne : la relation dialectique de cet Autre avec l'Être ; autrement dit le malentendu s'est édifié - et s'est perpétué - sur la méconnaissance du schème fondateur réglant la dynamique du discours philosophique en tant que discours philosophique 
et sa logique tensionnelle. Venant de convoquer au surgissement du philosopher, de l'acte de philosophie, Éros, le principe moteur de son déploiement, le démon qui relance son mouvement, je tenterai maintenant, avec son aide, de montrer qu'il est possible de faire cesser ce malentendu ayant engendré le conflit entre discours. Mais il me faut pour cela quitter le terrain où la guerre sévit pour retomber en deçà, à l'origine, ou plutôt, pour descendre en dessous - s'enfoncer dans son bathôs - en sonder l'bypokêimenon. Dès lors la question n'est plus celle de tout à l'heure : que fait la philosophie ? et le problème n'est plus de retrouver le schème de son fonctionnement, ce qui nous renseignait sur ses opérations spécifiques. La question qui se pose maintenant est celle de la condition originaire du philosopher, ou encore celle du schème lui-même, de sa possibilité ; et le problème consiste à identifier la source d'où le philosopher prend élan et mouvement, d'en caractériser la nature et les effets. Sachant qui est philosophie, peut-être ceci permettra-t-il de comprendre pourquoi le schème alors engendré revêt la forme de la dyade ; la généalogie du Logos philosophique nous livrerait ainsi le secret de son ressort. Ma thèse sera la suivante : Si, selon Platon, un " mythe » ressemble à la Vérité pourvu qu'on y voie un mythe et non la Vérité ${ }^{3}$, si, d'autre part, la catégorie " perspective féministe ressortit du "mythe ${ }^{4}$, et est revendiquée comme telle, je nommerai donc l'Être Philosophie Éros, et je m'inspirerai du mythe de sa naissance raconté par Diotime dans le Banquet pour caractériser le Logos philosophique comme la synthèse dialectique des opposés. Comme je n'oublie pas mon dessein final de conclure le traité de paix entre perspectives martiales, cette thèse qu'Éros en tant que synthèse vivante des opposés est Philosophie, devra être suivie d'une seconde thèse : je dois

3. Le mythe représente, sous une forme figurée et avec les procédés de la Fable, la doctrine positive. Platon emploie le mythe pour traduire ses idées sous une forme concrète. Il ne vaut pas par lui-même et il importe par conséquent de chercher à savoir quel est, dans toutes ses parties, le sens de l'allégorie employée.

4. Le "mythe " entendu ici au sens sorélien : dans ses Réflexions sur la violence Georges Sorel note l'efficacité des représentations idéologiques et des systèmes d'images qu'il appelle des mythes. "On peut, écrit-il dans ce texte, indéfiniment parler de révoltes sans provoquer jamais aucun mouvement révolutionnaire tant qu'il n'y a pas de mythes acceptés par les masses ". Et ce qui fait à ses yeux la force d'un mythe ", c'est qu'il ne saurait être réfuté puisqu'il est, au fond, identique aux convictions d'un groupe, qu'il est l'expression de ces convictions en langage de " mouvement". 
qualifier ces opposés tout en tenant compte, d'une part, de la nature de la relation qui les unit et des effets de celle-ci, de l'autre, du débat qui nous occupe aujourd'hui ; je soutiendrai donc qu'une des figures d'Éros Philosophe se dessine selon l'entrelacement de la Vie et de la Pensée, d'Anêr et de Gunê, Philosophie est Androgyne ; thèse à entendre abstraction faite de toute sexualisation. Les termes du rapport traditionnel entre Vie et Pensée, ou Sentiment et Raison, ou encore Monde Sensible et Monde Intelligible, peuvent sans empêchement, me semblet-il, être aussi énoncés, en fonction de la question en cause ici, et pour l'éclairer, Anêr et Gunê : d'une part parce que les réalités ou les notions auxquelles le rapport traditionnel fait référence, englobent ou subsument, et $j$ 'avance ceci d'après le texte platonicien lui-même, les traits ressortissants aux figures permanentes du Masculin et du Féminin ; je me suis donc crue suffisamment autorisée pour relier, sur le mode métaphorique, la sphère de l'Anêr à celle du monde intelligible et la sphère de la Gunê à celle du monde sensible, à exprimer à travers le couple Anêr/ Gunê l'analyse de la nature contradictoire de l'être philosophique et à jouer ainsi de la possibilité de ces correspondances et de ces enchaînements pour le développement de mes thèses. D'autre part, l'emploi de ces deux métaphores de la Vie et de la Pensée permet la mise en relief, sur le terrain du discours philosopbique, des caractérisations du masculin et du féminin ainsi que du prétendu clivage entre ces termes, telles que le discours polémique féministe - aux enjeux politico-idéologiques - reproche au discours philosophique d'établir.

Ni féminin, ni masculin, le logos philosophique, considéré sous l'aspect de la thèse de son androgynéité, est ensemble féminin et masculin. "Opérateur " de sa dynamique, Éros Androgyne engendre son mouvement et le dialectise : il l'ordonne selon le schème fondamental du Même et de l'Autre ; règle le cortège (la théorie, dirais-je, étymologiquement parlant) des dyades du discours philosophique historicisé comme autant de métaphores de sa double et pourtant unique essence.

3.2 Adressons-nous encore à Platon : cette fois pour déterminer avec lui la nature et les effets d'Éros. Dans le Banquet, une étrangère, mais qui ne commettra aucun parricide, Diotime, prêtresse de Mantinée, expose, après les cinq discours précé- 
dents, la théorie philosophique de l'Amour ${ }^{5}$. Le récit de la naissance de l'Amour nous replonge dans le mythe. La nature d'Éros est représentée comme une synthèse des caractères de ses deux géniteurs. Le père de l'Amour est Expédient (Poros), qui luimême est le fils de Sagesse ou Réflexion (Mêtis). Sa mère est Pauvreté (Pénia). Le développement qui suit déterminera la portée du mythe tout en explicitant les symboles qui y sont contenus. De même le portrait de Socrate répondra dans la dernière partie du Banquet, aux qualités d'Éros "expliquées " par sa naissance mythique; Socrate sera décrit par Alcibiade comme le véritable eroticôs, en même temps que le type vivant du philosophe. Platon insiste sur trois traits d'Éros : sa nature est synthétique - entièrement faite de contrastes solidaires, Éros est, par suite, double, contradictoire et instable dans son unité actuelle et dans son équilibre mouvant - médiatrice - caractère qui est également celui de l'âme et des démons. Enfin Éros est rapproché de la Dialectique : par sa nature intermédiaire, il est à la fois " un don qui vient de l'intelligible, un effort qui part du sensible » (L. Robin, op. cit. p. 176). Nous devons voir maintenant comment, de ces caractères de la nature d'Éros, on peut en induire mes deux thèses.

3.3 Éros est l'enfant de Pénia et de Poros. Il doit à son ascendance maternelle d'être tout l'opposé du délicat, du raffiné ; aucunement soucieux du luxe, du confortable (Banquet 203 d). À son ascendance paternelle il doit d'être toujours en quête de ce qui est beau et bon - le grand souci de tout citoyen athénien - ; d'être hardi, inventif ; viril, souligne Platon; passionné de savoir, c'est-à-dire acharné à philosopher « employé à philosopher toute sa vie " (Banquet $203 \mathrm{~d}$ ) ; sorcier, magicien aussi, créateur d'illusions à la façon d'un Sophiste. Tous ces traits donnent sa détermination statique, en quelque sorte. Sous son aspect dynamique, autrement dit dans son devenir et dans la façon dont Éros traduit et développe sa nature, celle-ci se révèle encore contradictoire, et n'étant ni d'un mortel, ni d'un immortel, ni savant ni ignorant, être intermédiaire, c'est pour tout

5. Cf. pour une analyse détaillée : L. Robin : La théorie platonicienne de l'amour ; PUF, 1964, nouvelle édition. Mon ambition ici n'est pas de faire une étude d'Éros mais de jouer avec Éros pour rappeler l'impulsion dialectique du discours philosophique. 
dire un « démon ${ }^{6}$ (Banquet, 203 e). Éros prend tour à tour le visage dû à l'hérédité paternelle, celui dû à l'ascendant maternel, si bien qu'en fin de compte, son opulence n'est pas plus essentielle et durable que ne l'est son dénuement.

Ce que Platon veut mettre en relief, et ce qu'il cherche à nous rendre sensible par le mythe, expression figurée de sa doctrine ${ }^{7}$, c'est la complexité de la nature duelle d'Éros, la synthèse de ses qualités contradictoires et l'instabilité de cette synthèse ; par suite, la tendance incessante à passer d'un état moins parfait à un état plus parfait. La conséquence la plus importante de cette synthèse indestructible et qui fait d'Érôs non pas un mixte hybride, mais une unité concrète et vivante de déterminations contraires, c'est, nous dit Platon, et par le fait même de son origine, qu'Amour est philosophe (Banquet 203 et sq.). Ce n'est pas là l'un des facteurs de sa complexion (le naturel de son père) qui l'emporte et qui domine, mais bien un effet de sa complexion synthétique, de la fusion en lui et par lui du naturel de sa mère et de son père. Éros est fils de Pénia et de Porôs c'est-à-dire selon les développements platoniciens - qu'il participe de la nature sensible à laquelle les Idées font défaut, et de la nature intelligente qui a connu les Intelligibles mais qui les a oubliés. C'est de cette manière qu'Éros est lié intimement à la philosophie, qui aspire à rejoindre le monde intelligible à partir du monde sensible, et que le philosophe, unité contradictoire de deux principes opposés, du Fini et de l'Infini, du savoir et de l'ignorance, est aussi le parfait amoureux.

Que l'Amour soit accueilli de façon privilégiée par la Philosophie, cette thèse se dégage encore de l'étroite liaison que Platon établit entre l'Amour et la personne de Socrate. Cette conciliation, dans le même homme, de l'Amour et de la Philosophie, sert à montrer que, par tous ses traits, le caractère du Socrate réel répond à la relation précédemment établie entre la

6. On se souviendra que dans plusieurs dialogues, notamment dans la République, le Théétète, le Sophiste, Platon qualifie le philosophe d'être intermédiaire, entre l'immortel et le mortel, entre le savoir et l'ignorance, quasi divin. Et Socrate, "être démoniaque ", lui-même, incarnation du philosophe, n'évoque-t-il pas souvent le démon qui le guide?

7. J'insiste encore une fois sur la valeur propédeutique de ce procédé chez Platon qui sert à la libération de l'intuition, de la " vision intellectuelle " de l'essence. 
définition de l'Amour et celle du vrai Philosophe ${ }^{8}$. D'abord le Socrate de Platon fait profession, à maintes reprises, non seulement dans le Banquet, ailleurs, de " ne savoir que l'amour ». ${ }^{9}$ Ensuite il a tous les caractères qui sont attribués à l'Amour. En premier lieu, il est philosophe, et l'Amour est philosophe, précisément parce qu'il unit en lui des traits opposés (Banquet $203 \mathrm{~d}$ et sq.) dont l'ignorance et la sagesse sont les plus caractéristiques ; pauvre, laid, Socrate va nu-pieds ; à l'affût des beaux objets, habile chasseur, il part à la recherche de l'essence pure des êtres. De même que l'Amour, Socrate possède une puissance magique, ses discours sont ceux d'un enchanteur ${ }^{10}$. Tout ce que dit Alcibiade de la nature contradictoire de Socrate et de l'effet de ses discours est l'application de ce qui a été dit plus haut sur l'Amour comme synthèse des opposés. Platon, avec intention, substitue dans la bouche d'Alcibiade, l'éloge de Socrate à l'éloge de l'Amour (Banquet $214 \mathrm{~d}$ ) : loc er Socrate, c'est encore louer l'Amour, car Socrate c'est l'Amor r lui-même et l'Amour, c'est la Philosophie, la prédominance dans une nature une de l'intelligence sur les sens, en vue de ! ı Beauté, but qui précisément accomplira l'unité de cette nature.

3.4 Cette identification d'Éros avec la Philosophie, dérivant de la nature synthétique d'Amour, dépend également de la nature intermédiaire de celui-ci. Cette seconde caractéristique est étroitement liée à la première : la nature synthétique d'Éros fait de lui un intermédiaire entre les qualités opposées que cette nature a pour fonction d'unir. La notion d'intermédiaire élargit ainsi la conception de la nature synthétique d'Éros. Elle suppose, en effet, que les termes dont Amour effectue la liaison sont des termes séparés et possédant une existence indépendante ; il s'ensuit qu'Éros représentera lui-même, à la fois, une réalité intermédiaire, et par l'union de cette réalité avec la nature de la philosophie, un moyen de passer de l'un à l'autre de ces termes.

L'idée d'Éros médiateur s'exprime dans le Banquet sous une forme bien connue : Amour est un démon et un grand démon.

8. "Il y a ainsi, écrit Brochard (in Ann. philos. XVII, p. 25) entre les deux parties du dialogue le même rapport qu'entre l'abstrait et le concret, la théorie et la pratique, le principe et l'application ".

9. Lysis 204 b-c ; Phèdre 227 c ; 257 a.

10. Â plusieurs reprises, dans le Cbarmide, le Ménon, le Phédon, le Théétète, le Philosophe est présenté comme un enchanteur. 
Or la fonction des démons est d'être les intermédiaires entre les immortels et les mortels, d'unir l'une à l'autre les deux sphères, de remplir l'intervalle qui les sépare, de donner à l'Univers l'unité et la liaison (Banquet : 202 e). Unissant, on l'a vu, par le désir philosophique du savoir, le domaine du sensible et celui de l'intelligible, la connaissance et le défaut de connaissance, la part de sa nature de ce qui est appelé par Platon, virile, et la part qui en est féminine, Éros, en tant que démon, est un intermédiaire entre le monde d'ici-bas (le Multiple) et le monde supérieur (l'Un), entre le Sentiment et la Raison, l'Anêr et le Gunê. Diotime qui, non moins savante en amour, a appris à Socrate tout ce qu'il sait à ce sujet (Banquet 207 d) a précisément pour rôle, dans le dialogue, de faire apparaître la vision d'une réalité distincte de celles ressortissant aux catégories de l'expérience commune et affranchie des conditions qui font cellesci multiples, changeantes et périssables (Banquet, $210 \mathrm{a}-212 \mathrm{~b}$ ). Diotime, la femme inspirée, est donc, comme Éros dont elle discourt, un être intermédiaire ; un être démoniaque. Et le portrait de Socrate que dresse son amant Alcibiade est aussi celui d'un être démoniaque, à la nature équivoque et contradictoire, ressemblant moins à un homme qu'à un de ces démons, Silènes ou Satyres (Banquet 215 b-221 d), être " atopique " qu'on ee peut placer nulle part. Éros Intermédiaire qui unit les êtres, qui établit une communion entre la terre et le ciel, est inséparable de ce qu'il a pour fonction de mouvoir ; étant lui-même automoteur, il constitue une relation perpétuellement mouvante entre le Devenir et l'Être, entre le Sensible et l'Intelligible, entre la Vie et la Pensée, bref entre les sphères dévolues culturellement (ce que le discours féministe ne désavouera guère) à l'Anêr et à la Gunê : son rôle révèle la signification symbolique de sa nature d'intermédiaire, de sa nature démoniaque, Éros est Androgyne.

Les commentateurs ont vu dans la doctrine platonicienne de l'Amour comme être intermédiaire entre les réalités une solution indirecte au problème de la participation. Si Éros est un intermédiaire à l'aide duquel la sphère du Sensible et celle de l'Intelligible sont réconciliées, il est clair que les étapes de cette médiation seront, inversement, les moyens termes de la Participation. La réalité qu'Éros Philosophe aspire, par essence, à posséder éternellement, le Beau, lié au Bien et au Vrai, se répand 
en des termes qui en sont des expressions, telles par exemple l'Anêr et la Gunê, graduellement affaiblies, mais non point opposées ; chacune de ces expressions représente un des moments par lesquels il faudra au contraire passer, si partant d'en bas, le philosophe veut retourner vers la réalité absolue. Il n'y a donc pas rupture complète entre les sphères opposées ni non plus identification complète (monisme) ou absorption de l'une par l'autre.

La doctrine platonicienne de la participation dont, comme je l'ai avancé tantôt, Éros Androgyne (Intermédiaire) serait une expression symbolique, peut se présenter encore à un autre point de vue. On y signalera la trace d'un des grands mythes de la culture occidentale, le mythe fusionnel, le mythe de la bisexualité se déposant ainsi dans l'armature du discours philosophique à ses origines. Et Platon, en reprenant de la sorte ce mythe, ferait pièce au mythe rival, celui de la séparation, de la distance entre l'homme et la femme. La figure d'Éros (Intermédiaire) Androgyne, Masculin-Féminin, Sentiment-Raison, se souvient, dira-t-on, de l'existence sur le bassin méditerranéen des dieux hermaphrodites - Dionysos vêtu de la longue robe de femme - ou du prêtre syrien qui relevait d'un geste sacré l'anasyrma, sa robe féminine sur un phallus dressé ${ }^{11}$, et, dans un registre voisin, des femmes à fonction masculine : prêtresses inspirées telles Diotime elle-même, les Sibylles ou les Pythies qui incarnent de façon croisée l'homme dans la femme.

Hegel, l'héritier de l'intuition dialectique platonicienne, fera lui aussi apparaître à deux reprises, dans la Phénoménologie et dans la Philosophie du Droit, les marques du mythe fusionnel imprégnant la culture philosophique. Deux personnages à la fois tragiques et logiques, Antigone et Électre, en feront la mimêsis. Analysant le rapport d'Électre à son frère Oreste, avec qui elle fait corps pour obtenir la vengeance contre leur mère Clytenestre meurtrière d'Agamemnon, son époux, Hegel écrit "le frère est pour la sœur l'essence apaisée et égale en général . . . le frère perdu est pour la sœur irremplaçable . . . » Le philosophe féministe Catherine Clément commente ainsi la représentation hégé-

11. Cf. les ouvrages de J.P. Vernant et notamment : Mythe et Pensée chez les Grecs. Maspéro, 1965 . 
lienne de la fusion. Je cite à peu près : " c'est une relation sans mélange : parfaite parce qu'elle échappe à l'inceste physique tout en assurant l'idéal fusionnel : la réunion du même - il s'agit du même sang - et de l'autre - il s'agit des deux sexes. Mais la séparation interviendra agencée néanmoins à la fusion, par la répartition des fonctions logiques entre homme et femme : du côté de la femme se trouvera l'inconscient, la divinité, le singulier, la capacité d'enterrer le mort, et de boucler ainsi la boucle de la vie et de la mort ; du côté de l'homme se trouvera le conscient, l'état civil humain, l'universel, la guerre. Dans La philosophie du Droit, Hegel mettant en forme l'idée de famille, décrira Antigone (\# 166) comme synthèse-tragique - entre la Femme et l'Homme. Femme, Antigone l'est essentiellement puisqu'elle n'apparaît dans l'Histoire que comme une sœur qui veut enterrer son frère. Mais pour le faire elle transgresse et innove, elle prend un rôle d'homme comme le souligne - et le lui reproche - sa sœur Ismène. Et passant de l'intériorité - le foyer, l'inconscient, la rumination close - à l'extériorité - le dehors, la conscience entêtée, l'acte politique - Antigone réunit en elle la féminité et la virilité. Elle est, logiquement, le «pour-soi " masculin et l'« en-soi "féminin.

3.5 Éros est un démon, il établit une relation entre des mondes opposés, il constitue, lui-même, cette relation, cette union de la nature sensible à laquelle participe la Gunê et de la nature intelligible à laquelle participe l'Anêr ; mais à ce titre Éros Androgyne (Intermédiaire) est un succédané de la Dialectique : il apparaît maintenant comme le moyen d'opérer le passage entre mondes opposés, le moyen d'acquérir et de communiquer la vertu, le moyen d'acquérir et de communiquer la science, ce qui est la « raison d'être de la Dialectique et de toute vraie philosophie ». La nature d'Éros se relie ainsi à la tâche proprement philosophique.

Éros n'est pas un intermédiaire fixe, comme le serait par exemple l'opinion entre l'ignorance et la science. Sa nature est telle qu'il tend sans cesse à unir les extrêmes, à les concilier l'un avec l'autre (on se retrouve ici devant l'insistance platonicienne sur le mouvement qui lie les opposés et de ce fait réalise leur union, les prémices de l'axiome de la dialectique moderne : le primat de la contradiction sur les contraires). De plus Éros est 
ce que n'est pas l'opinion car il constitue par lui-même une méthode, c'est-à-dire une transition, au sens propre du mot, un passage, un mouvement vers un but auquel il atteint sans cesser d'être ce qu'il est. C'est pourquoị l'Amour sera justement nommé philosophe, aux termes mêmes de l'analyse du $\mathrm{V}^{\mathrm{e}}$ livre de la République. La synthèse de contraires qui constitue Éros - le viril et le féminin étant deux de ces contraires, ne l'oublions pas - est une véritable synthèse. Comme l'écrit $\mathrm{L}$. Robin (op. cit.) : « ce n'est pas seulement une pièce en quelque sorte intercalaire ; c'est un principe de jonction ou d'union, tel que l'un des termes du couple d'opposés participe grâce à lui de l'autre terme ", tel que le sensible ne reste pas étranger éternellement à l'Intelligible ou encore l'Anêr à la Gunê.

On le sait c'est l'influence d'Héraclite qui détermina Platon à modifier par sa théorie des contraires, sans faire tort au principe de contradiction ${ }^{12}$, l'intellectualisme parménidien. Le principe héraclitéen de l'alternance des contraires se retrouve ici adapté dans le Banquet aux exigences de la théorie platonicienne de l'amour ainsi qu'à celles de la correspondance entre Éros et Dialectique. L'impulsion philosophique est celle de la possession de la vérité mais c'est l'art dialectique qui permet d'atteindre cette possession. Éros est Dialectique en ce sens qu'il constitue la voie qui mène du Non-Être à l'Être, qu'il incarne (représente) la méthode dialectique propre au philosopha : Éros demande à l'âme raisonnable de chercher pour ainsi dire jusque dans la « pauvreté » de sa nature mortelle un moyen d'obtenir la conversion vers les Intelligibles. Éros Dialectique utilisera une émotion particulière de l'âme mortelle pour détourner l'âme immortelle vers la connaissance et lui permettre de contempler, par les moyens qui lui sont propres, le Beau en lui-même et avec lui les Intelligibles qui sont son objet naturel.

Érôs se lie alors à la Maïeutique afin de faire parvenir le philosophe à la connaissance de l'essence. Éros a été défini comme " procréation dans la beauté selon le corps et selon l'âme ». Cette puissance de créer qui définit sa fonction, au point de vue dynamiste, se manifestant au voisinage de la Beauté, produira dès

12. Cf. Phédon, 103 a-c; Sophiste 256 d-257 a ; 258 d-259 a, l'Être est à la fois et mouvement et repos. 
lors les dyades qui règlent le discours philosophique et qui sont autant de métonymies discursives de sa nature duelle et contradictoire que les fruits de la dialectique. Le Même et l'Autre, l'Être et le Non-Être, l'Un et le Multiple, le Grand et le Petit, l'Ordre et le Désordre, le Fini et l'Infini pour ne mentionner que quelques-unes des dyades du logos philosophique païen. Et s'il est permis de faire reculer l'horizon du Banquet on retrouvera les structures dyadiques parmi celles qui constituent le paradigme philosophique de l'occident moderne : sujet-objet ; continu-discontinu ; ouvert-fermé . . . et dans le binarisme du discours contemporain : celui du signifiant-signifié, du savoir et du non-savoir, du centre et de la périphérie. Chacune de ces dyades nè se forme et ne se développe que dans le mouvement même de la fécondation érotico-dialectique leur permettant le rapport à deux termes, où le primat de l'un ou de l'autre s'épuise dans une rivalité dérisoire. Selon cette manière d'être dyadique se constitue, se déploie et se renoue ainsi à elle-même l'indéchirable unité du réel philosophique.

\section{LES MAMELLES DE TIRÉSIAS.}

Les aspects et les moments de la réalité tendent, il est vrai, à se dissocier, à s'affirmer chacun pour soi, selon les déterminations de la conjoncture politico-idéologique, en s'autonomisant. D'où la séduction peut-être inévitable des perspectives féministes ou autres qui, suivant cette fragmentation incessante de la réalité, polarisent leur interprétation en isolant l'une de ses facettes. En proposant mes deux thèses je ne visais pas tant à faire éclater une de ces monades, à abolir la perspective féministe sur la philosophie, qu'à surtout tenter une entreprise irénique: à illustrer par cette esquisse d'une perspective hermaphrodite, le lieu de la " coïncidence des opposés ", le soubassement du champ philosophique ; et encore, à faire apparaître l'impulsion dialectique du discours philosophique. L'acoustique du Banquet a permis d'entendre les harmoniques reliant Éros à Philosophie : unité des contraires, démon androgyne, dialectique. On peut conclure avec Léon Robin qui, au terme de son ouvrage sur la théorie platonicienne de l'amour, s'exprime ainsi: . . . « Éros apparaît (. . .) la loi universelle qui anime tout le réel, qui fait vivre la nature, qui meut l'âme du monde, qui lie 
dans l'Intellect l'Intelligible et le Sensible, qui opère dans le monde idéal le mélange des termes et les suspend enfin au Bien, sous son triple aspect de la proportion, de la beauté et de la vérité " (op. cit. p. 189). Une synthèse incessamment renouvelée de contraires, réglée en vue du Vrai et du Bien, voilà ce que serait enfin et telle qu'elle enveloppe ma vision, ÉrosPhilosophe-Androgyne. Et pourquoi ne pas imaginer comme Rilke l'écrivait à un jeune poète que :

. . . Les sexes sont plus parents qu'on ne le croit ; et le grand renouvellement du monde tiendra sans doute en ceci : l'homme et la femme libérés de tous faux sentiments, de toute aversion, ne se rechercheront plus comme des contraires, mais comme des frères et sœurs, comme des proches. Ils uniront leur humanité ...

R.M. Rilke

Lettres à un jeune poète. 